\title{
Nanotechnology intervention of the microbiome for cancer therapy
}

\author{
Wantong Song ${ }^{(1}$, Aaron C. Anselmo ${ }^{2 \star}$ and Leaf Huang ${ }^{2,3 \star}$
}

The microbiome is emerging as a key player and driver of cancer. Traditional modalities to manipulate the microbiome (for example, antibiotics, probiotics and microbiota transplants) have been shown to improve efficacy of cancer therapies in some cases, but issues such as collateral damage to the commensal microbiota and consistency of these approaches motivates efforts towards developing new technologies specifically designed for the microbiome-cancer interface. Considering the success of nanotechnology in transforming cancer diagnostics and treatment, nanotechnologies capable of manipulating interactions that occur across microscopic and molecular length scales in the microbiome and the tumour microenvironment have the potential to provide innovative strategies for cancer treatment. As such, opportunities at the intersection of nanotechnology, the microbiome and cancer are massive. In this Review, we highlight key opportunistic areas for applying nanotechnologies towards manipulating the microbiome for the treatment of cancer, give an overview of seminal work and discuss future challenges and our perspective on this emerging area.

T he microbiome, defined as the collection of the microorganisms and their genetic material in a particular environment, has long been implicated to play important roles in human health and disease ${ }^{1}$. It has been demonstrated that specific bacteria, and also the balance of bacteria in the microbiome, can contribute to carcinogenesis through various paths including bacteriaderived carcinogens, inflammation caused by bacterial infection or modulation of the immune response caused by dysbiosis ${ }^{2,3}$ (Fig. 1a). Helicobacter pylori (H. pylori) has been strongly linked to stomach cancer via type IV secretion of bacteria-derived carcinogens ${ }^{4}$ (Fig. 1a (i)). Fusobacterium nucleatum (F. nucleatum) has been linked to colorectal cancer through their adherence and invasion into intestinal epithelial cells, followed by $\beta$-catenin signalling, which leads to increased oncogenic and inflammatory responses ${ }^{5}$ (Fig. 1a (ii)). Certain carcinogenic Escherichia coli (E. coli) strains cause inflammation and are specifically expanded at sites of inflammation; inflammation can affect microbiota composition which in turn promotes carcinogenesis ${ }^{6}$ (Fig. 1a (iii)). These specific mechanisms (that is, bacteria-derived carcinogens, cell-signalling and inflammation), and others (that is, reactive oxygen species, DNA damage and increased gut permeability) are responsible for bacteria or microbiome-initiated carcinogenesis and have been previously reviewed in detail ${ }^{3,7}$. Importantly, the role of the microbiome in cancer extends beyond both the primary tumour site and the host's gastrointestinal (gut) microbiome, since specific cancer-causing bacteria are often detected in distal tumour sites such as metastatic lymph nodes and liver metastases ${ }^{8}$ (Fig. 1b). For the purpose of this article, we define bacteria found in distal tumours/metastases as tumour-associated bacteria (TAB). Additional efforts have described how microbiome or bacterial metabolites originating in the gut, such as bacteriaderived carcinogens or toxins, can initiate or affect non-gut cancers (for example, in the breast) ${ }^{2,3}$ and how bacterial species in the gut microbiome can colonize distal tumours (for example, in the pancreas) to affect patient survival ${ }^{9}$.
Beyond carcinogenic effects, considerable evidence indicates that the gut microbiome can enable or alter the efficacy of chemotherapies and immunotherapies. The chemotherapeutic cyclophosphamide relies partly on alteration of the microbiota (defined as the microorganisms that comprise the microbiome) and subsequent translocation of Gram-positive bacteria to secondary lymphoid organs to stimulate T-cell based immune responses ${ }^{10}$. In other examples, microbiota disruption via broad-spectrum antibiotics has been shown to negatively impact cancer immunotherapy outcomes $^{11}$ even in clinical studies ${ }^{12}$, highlighting the importance of the commensal microbiota in regulating immune response for cancer therapy. Building on these findings that connect the gut microbiome to efficacy of cancer therapeutics, recent preclinical work has demonstrated that microbiome modulation through administration and subsequent gut colonization of commensal bacteria (for example, Bacteroidales, Bifidobacterium, Akkermansia muciniphila) can enable and increase the efficacy of blockade therapies (that is, CTLA-4, PD-L1 ${ }^{13-16}$. Extending the role of bacteria in cancer therapy to $\mathrm{TAB}$, it was demonstrated that bacteria found in pancreatic tumours can mediate chemotherapy resistance by metabolizing gemcitabine into its inactive form ${ }^{17}$. Over ten different bacterial species demonstrated this effect against gemcitabine, including bacteria derived from primary pancreatic tumours ${ }^{17}$, indicating that TAB, independent of their role in cancer, can actively inhibit and induce resistance against widely used chemotherapies. Collectively, the above findings have created great interest in developing approaches to modulate the microbiome to remove cancer-causing bacteria or improve cancer treatment through addition of beneficial species, with many of these efforts having already entered clinical trials ${ }^{18,19}$.

Current approaches for microbiome modulation (for example, antibiotics, faecal microbiota transplants, probiotics, diet modifications and prebiotics) lack specificity in achieving targeted modulation, were not originally developed with the tumour microenvironment in mind, and may not have the ability to interact with 


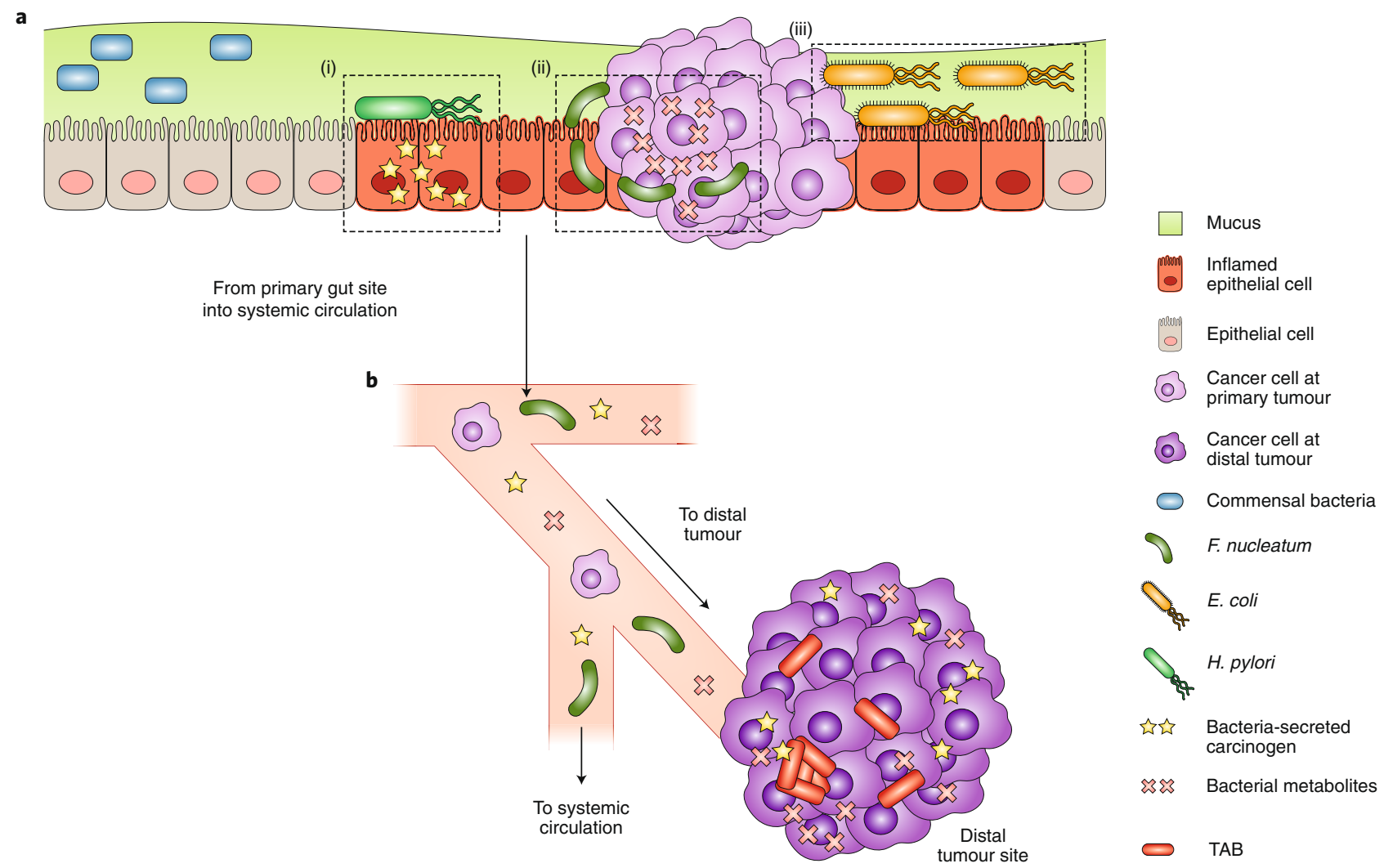

Fig. 1 | The role of bacteria and the microbiome in the tumour microenvironment. a, Examples of mechanisms of bacteria- or microbiome-initiated carcinogenesis in the gut: (i) type IV secretion of carcinogens by H. pylori, (ii) adherence and invasion of $F$. nucleatum into epithelial cells followed by oncogenic and inflammatory responses, and (iii) inflammation caused by E. coli, which can affect microbiota composition and promote carcinogenesis. b. Bacteria from the primary tumour can be found in distal tumour sites and influence metastasis. We have defined these bacteria as tumour-associated bacteria (TAB).

microbes that are not accessible through oral delivery routes such as $\mathrm{TAB}^{20-22}$. As such, new approaches for microbiome intervention geared towards cancer therapy must be able to: (1) navigate the complex microenvironment (encompassing the microbiome, the tumour microenvironment and the barriers at the tumour-microbiome interface), (2) specifically interfere with the responsible molecular pathways and (3) be functional at sites beyond the primary tumour (for example, lymph nodes and metastases). Nanotechnologies are positioned to potentially meet these diverse requirements as they are able to communicate across molecular and macroscopic length scales, a key requirement for interactions with small molecule metabolites, microscopic bacteria and macroscopic tumours/organs. While the application of nanotechnology to microbiome modulation for cancer prevention/treatment is still in its infancy, there exists seminal work that highlights its massive potential. This potential first relies on identifying which bacterial species are beneficial for cancer treatment ${ }^{19,23}$ versus harmful in cancer initiation and progression ${ }^{3,7}$; this is beyond the scope of this Review, as this has been covered extensively in previous reviews $^{3,7,19,23}$. In this Review, we discuss how the properties of nanotechnologies make them uniquely suited for microbiome intervention. We then highlight seminal work where nanotechnologies have been used to improve cancer therapy through interference with bacteria-derived carcinogens or through microbiome modulation. We also highlight an emerging area of research wherein unique functions of microbes, the microbiome or microbe communities can be used as a source of inspiration for improving traditional nanoparticle delivery. Finally, we share our perspective on the challenges and outlook of the application of nanotechnologies towards cancer treatment through microbiome interventions.

\section{Nanotechnology for microbiome intervention}

Nanotechnologies have been widely investigated for cancer treatment for the past three decades. The first-generation nanotechnologies have been approved for clinical use $\mathrm{e}^{24}$ and leverage the ability of nanotechnologies to navigate the vasculature ${ }^{25}$, avoid opsonization through hydrophilic coatings ${ }^{26}$, accumulate at tumours via the enhanced permeability and retention effect ${ }^{27}$, and control the release of the encapsulated $\mathrm{drug}^{28}$. Second-generation nanotechnologies are currently being investigated in ongoing clinical tri$\mathrm{als}^{29}$ and build on the existing advantages of nanotechnologies to add functions such as tissue targeting ${ }^{30}$, combination delivery ${ }^{31}$ and stimuli-responsiveness ${ }^{32}$. Additional innovations have led to the development of third generation nanotechnologies with even more functions such as immune system modulation ${ }^{33}$, biological barrier penetration ${ }^{34}$, and 'self-recognition ${ }^{35,36}$. Development of nanotechnologies through these generations has been enabled by an existing and ever-expanding nanotechnology toolbox ${ }^{30,32,37-39}$ that includes surface modifications (Fig. 2a), encapsulation and controlled release approaches (Fig. 2b), and engineering and modification of physical properties (Fig. 2c). This existing nanotechnology toolbox can be used to design nanoparticle systems specific for microbiome intervention in cancer (Fig. 2d); however, this represents a generally unexplored area.

The gut microbiome is an exceedingly complex environment that comprises trillions of microbes and resides at an interface 


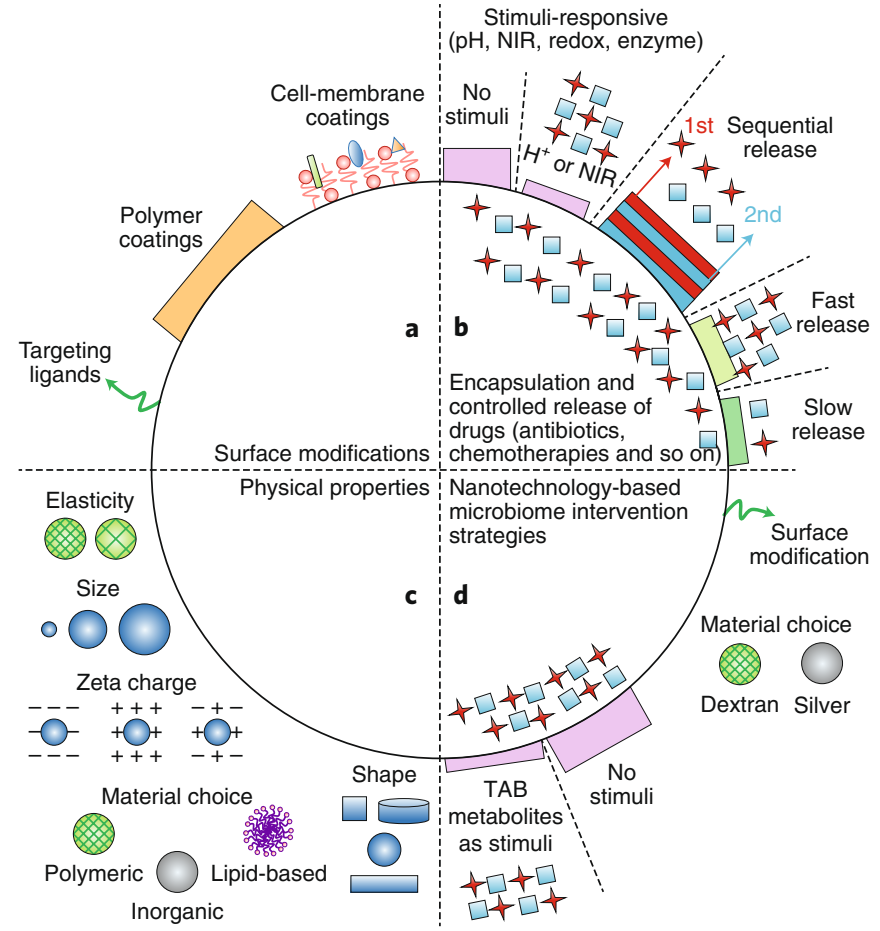

Fig. 2 | The current nanotechnology toolbox can be applied for microbiome intervention strategies. a-c, Current strategies for expanding the functionalities of nanotechnologies include surface modification (a), encapsulation and controlled release (b) and modulation of physical properties (c). d, These existing approaches could be used for microbiome intervention in cancer therapy. Surface modification strategies, such as ligand functionalization, can be used to target nanotechnologies to tumours, TAB or TAB metabolites. Physicochemical properties of nanoparticles are governed by material choice, which affects nanoparticlemediated toxicity against cancer-causing bacteria or the proliferation of anti-cancer commensal bacteria. Encapsulation and controlled release strategies can be used to deliver combinations of antibiotics or chemotherapeutics that can be released in response to local stimuli such as bacterial metabolites. NIR, near infrared.

consisting of the mucus barrier, the lumen and its contents (for example, food, water, mammalian cell secretions such as bile acids and microbe metabolites), and the epithelial cell lining in our gut ${ }^{40}$ (Fig. 1a). Based on the success of cancer nanotechnologies, we believe the existing nanotechnology toolbox can be leveraged to design nanotechnologies to navigate the microbiome microenvironment (Fig. 2d) since individual examples exist for nanotechnology targeting to the gut ${ }^{41}$, delivery to inflammation ${ }^{42}$, penetration and diffusion across mucus ${ }^{43}$, targeting to specific microbes ${ }^{44}$ and transport across the epithelium into systemic circulation ${ }^{45}$. Beyond intervention at the gut microbiome-tumour microenvironment, nanotechnologies can potentially target and act on migratory $\mathrm{TAB}$ and their metabolites, which persist in distal tumours 8,9 (Fig. 1b). The opportunities for targeting TAB with nanotechnologies arises from specific features of non-gut-associated tumour microenvironments. For example, many bacteria favour the hypoxic regions of tumours ${ }^{46}$ and nanotechnologies have demonstrated success in interacting with and delivering payloads to hypoxic regions $s^{47}$. TAB persistence in tumours may additionally arise from avoidance of immune-cell clearance in the immunosuppressive tumour environment, protective interactions with tumour-associated macrophages or the commensal relationship TABs share with tumour cells. Nanotechnologies can potentially intervene with persistent $\mathrm{TAB}$ or TAB-cell interactions as they have been previously utilized to enable immunotherapy by interacting with specific cell populations in the tumour ${ }^{48}$. As such, interventional approaches of the microbiome for cancer therapy exist at both the gut microbiome-tumour microenvironment and at distal sites of metastasis or tissues harbouring TAB. In any case, robust multifunctional nanotechnologies that can navigate a variety of biological barriers and interact with distinct cell types is required. Although nanotechnology navigation through systemic circulation and tumour vasculature is an important prerequisite for targeting TABs or their metabolites, circulation and vascular navigation of nanotechnologies has been extensively reviewed previously ${ }^{25}$. In the following sections, we will highlight specific examples wherein nanotechnologies were used to act at the microbiome-tumour microenvironment or on TABs and their metabolites at a vascular-accessible tumour site to enable or facilitate cancer therapy.

\section{Nanotechnology for manipulating microbiome metabolites}

The crosstalk between the microbiota, their metabolites and the immune system at the gut interface or at metastatic tumours can have profound effects on carcinogenesis and cancer treatment. In two extreme examples of bacteria-secreted products, toxins (for example, cytolethal distending toxin, Bacteroides fragilis toxin or colibactin) can directly induce DNA damage responses ${ }^{3}$, whereas short-chain fatty acids (for example, butyrate) secreted by anaerobic bacteria have shown to be tumour suppressive through upregulation of $\mathrm{p} 21^{49}$. In downstream mechanisms, pathogenassociated molecular patterns such as lipopolysaccharide (LPS) promote inflammation, affecting the immune system and modulating responses to systemic immunotherapies ${ }^{3,19}$. Many of these bacterial metabolites (Fig. 3a) and their roles in cancer are unknown and more efforts focused on uncovering mechanisms are needed ${ }^{50}$. By focusing on what is known, opportunities exist for nanotechnologies to manipulate microbial signals/metabolites and prevent their interactions with mammalian cells (Fig. 3). By interfering with harmful microbial-host cell interactions, nanotechnologies could be used for cancer prevention, as new therapeutic modalities, or to facilitate enhanced efficacy of existing therapeutic approaches (for example, immunotherapies). These nanotechnologies can potentially be used to interact with bacterial secreted products (Fig. 3a) that include toxins, carcinogens and immunosuppressive agents in the gut (Fig. 3b), in the blood stream (Fig. 3c), or in primary/metastatic tumours (Fig. 3d). Potential approaches to manipulate microbial products include nanotechnologies that can directly block, bind or otherwise inactivate these bacterial products (Fig. 3b(i),c(i),d(i)). Another approach may be nanotechnologies designed to secrete deactivating agents (Fig. 3b(ii),c(ii),d(ii)). Approaches that involve using nanotechnologies as a vehicle to genetically engineer mammalian cells to secrete these same deactivating agents (Fig. 3d(iii)) have already been described ${ }^{51}$. In addition to inactivating bacterial metabolites, nanotechnologies can be designed to release chemotherapeutic agents upon interaction with secreted bacterial products in the tumour microenvironment or the microbiome. Below, we will highlight a seminal example wherein a nanotechnology was used to interrupt the chemical communication between bacteria metabolites and the immune system to improve immunotherapy. We will then discuss a second example where targeting bacterial metabolism was used to enable stimuli responsive drug release of a chemotherapeutic from a nanoparticle to facilitate killing of tumours with TAB burdens.

LPS, a major cell wall component of gram-negative bacteria, activates toll-like receptor 4 (TLR4), nuclear factor- $\mathrm{\kappa B}$ (NF- $\mathrm{\kappa B}$ ) pathways and transcription 3 (STAT3), which are heavily implicated in epithelial carcinogenesis ${ }^{3}$. Specifically, LPS-initiated activation of oncogenes has been strongly linked to colorectal cancer (CRC) and other cancers $^{52}$. Our lab has recently shown that a nanotechnology-based 

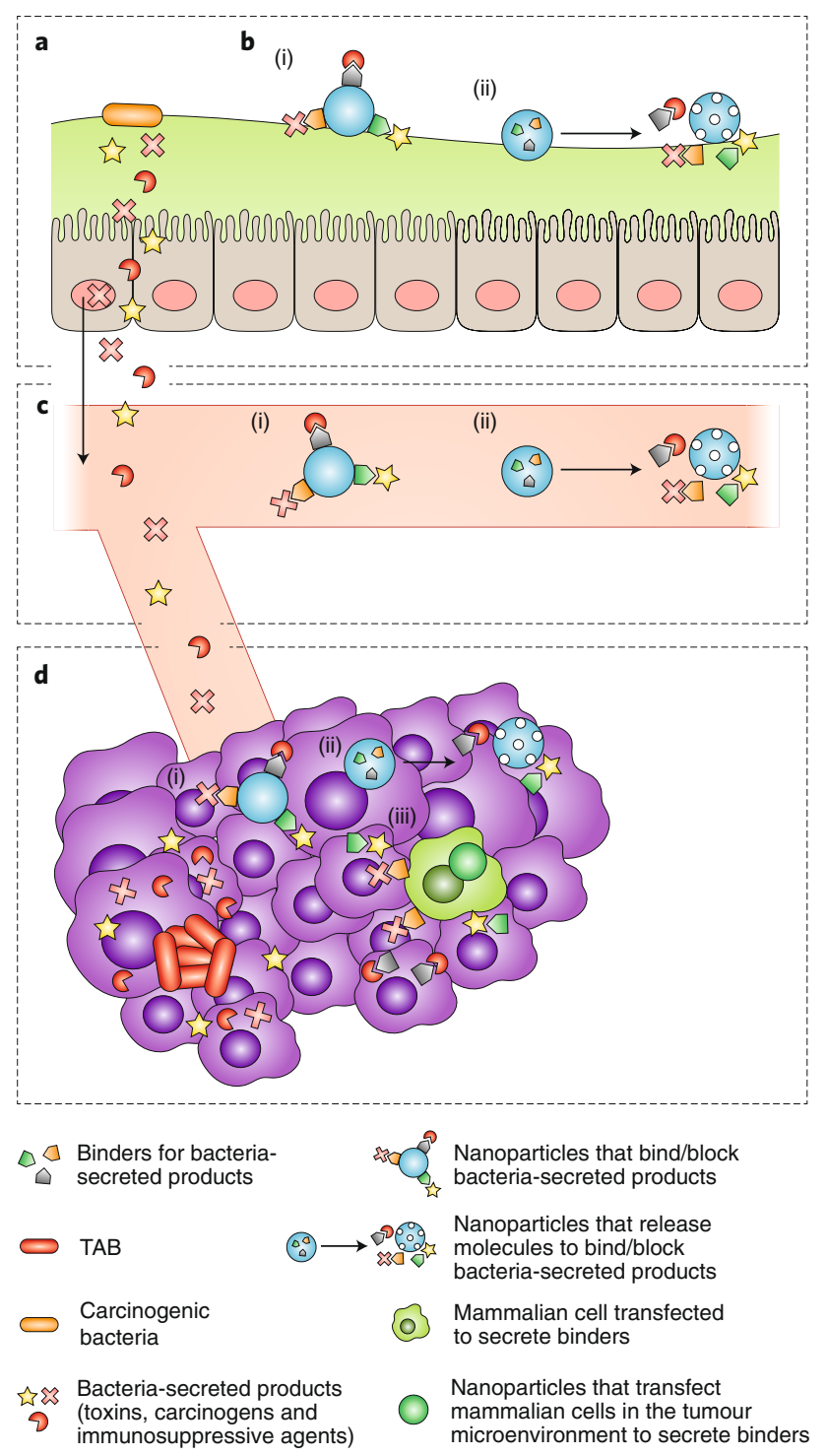

Fig. 3 | Nanotechnology to interfere with microbiome signals/metabolites. a, Potential application of using nanotechnologies for interference with bacterial metabolites such as toxins, immunosuppressive agents and carcinogens. b-d, Nanotechnologies in the gut (b), the vascular system (c), or tumours (d) can be used to (i) bind or (ii) secrete binders against bacterial metabolites. Nanotechnologies can also transfect mammalian cells in the tumour microenvironment to secrete binders (iii).

LPS trap protein improves efficacy of immunotherapies by blocking LPS-TLR4 binding, subsequently preventing oncogene activation. In this work, orthotopic and subcutaneous CRC tumours were separately established in mice and, in comparison to the subcutaneous model, the orthotopic tumour in the gut was: (1) in close proximity to the gut microbiome, (2) non-responsive to antiPD-L1 immunotherapy and (3) exhibited decreased T-cell infiltration. Analysis of LPS in both the orthotopic and subcutaneous tumours revealed that the orthotopic tumours contained over 60 -fold more LPS. Clearance of gram-negative bacteria from the gut via oral antibiotics against gram-negative bacteria (polymyxin B) decreased LPS, restored T-cell infiltration and reversed the immunosuppressive microenvironment in the orthotopic tumour. Using our existing lipid-protamine-DNA nanoparticle gene delivery system ${ }^{53}$, we encapsulated a plasmid that transfects mammalian cells to secrete an LPS-binding fusion protein (LPS-trap) (Fig. 4a). Systemic delivery of LPS-trap nanoparticles to the orthotopic tumour facilitated mammalian cell transfection, secretion of LPS-trap, and blocking of LPSTLR4 binding in the tumour microenvironment. Treatment with our nanotechnology resulted in increased T-cell infiltration in the orthotopic tumour and significantly decreased tumour growth as compared to anti-PD-L1 therapy alone. The efficacy of our nanotechnology in decreasing tumour burden was further improved by over 5-fold when used in combination with anti-PD-L1 therapy, as compared to the LPS-trap alone ${ }^{51}$. This study provides compelling evidence towards leveraging existing advantages of nanotechnologies (for example, gene editing, tumour accumulation and tumour delivery) to control microbiome-tumour interactions for increasing the efficacy of traditional immunotherapy.

Bacteria that colonize primary tumours in the colon can potentially breach the intestinal epithelium to eventually persist in sites of metastasis and satellite tumours as TAB. For example, F. nucleatum, the most prevalent bacteria in CRC tumours ${ }^{54}$ is frequently found in distal liver metastases ${ }^{8}$ and bacteria from the microbiome can migrate to the pancreas through the pancreatic duct ${ }^{55}$. In these cases, TAB have been known to influence inflammation, immune response and resistance to chemotherapeutics ${ }^{5,56}$. As such, TAB and their metabolites represent a potential target for intervention that can distinguish the tumour from healthy tissues. A recent study highlighted how metabolites secreted by TAB catalyze degradation of a doxorubicin-loaded nanoparticle for on-demand and stimuli responsive drug release at the tumour site (Fig. 4b). Specifically, a triple-layered polyethylene glycol/poly( $\varepsilon$-caprolactone)/polyphosphoester nanogel (TLND) was designed to degrade in the presence of bacterial lipase and subsequently release encapsulated doxorubicin into the tumour microenvironment. It was confirmed in vitro that triggered doxorubicin release induced cytotoxicity against $\mathrm{H} 22$ hepatoma cells and that release occurred only in the presence of bacterial lipase. A hepatoma xenograft model infected with lipasesecreting Staphylococcus aureus was developed to mimic a solid tumour harbouring TAB. Systemic administration of TLND to this model decreased tumour weight over 2 -fold, as compared to standard doxorubicin treatment, and over 4-fold as compared to TLND treatment in the absence of TAB burden ${ }^{57}$. These results highlight the potential of designing nanotechnologies to respond to a single bacterial metabolite in the tumour microenvironment to enable a new treatment modality of stimuli-responsive and on-demand release of anti-cancer drugs mediated by bacterial metabolism.

\section{Nanotechnology for microbiome modulation}

Individual bacteria and the collective behaviour of the microbiome play specific roles in initiating cancer. Strategies to alter the microbiome composition towards protection from, or treatment of, cancer can include addition of beneficial bacterial species, deletion of cancer-causing bacterial species or modulation of the existing commensal population ${ }^{22}$ to promote the proliferation of beneficial anti-cancer bacterial species such as those that secrete the short chain fatty acid butyrate ${ }^{58,59}$. Nanotechnologies for microbiome modulation have been for these latter two scenarios. Antibiotics are routinely used for killing pathogenic bacteria and modulating the microbiome; however, the use of broad-spectrum antibiotics can lead to antimicrobial resistance ${ }^{60}$, dramatic changes to the commensal microbiome which have been shown to decrease immunotherapy efficacy ${ }^{11}$ and initiation of dysbiosis and/or inflammation ${ }^{61}$, which are linked to cancer ${ }^{62,63}$. Nanotechnologies have been used to load and deliver antibiotics to kill bacteria, including cancercausing bacteria ${ }^{64,65}$. By virtue of properties that arise at the nanoscale, inorganic nanoparticles that inherently have antimicrobial activity have been used to kill cancer-causing bacteria ${ }^{66,67}$, primarily through generation of reactive oxygen species ${ }^{68}$. For both of these classes of antimicrobial nanotechnologies, the majority are nonspecific and were not designed to selectively kill individual species 


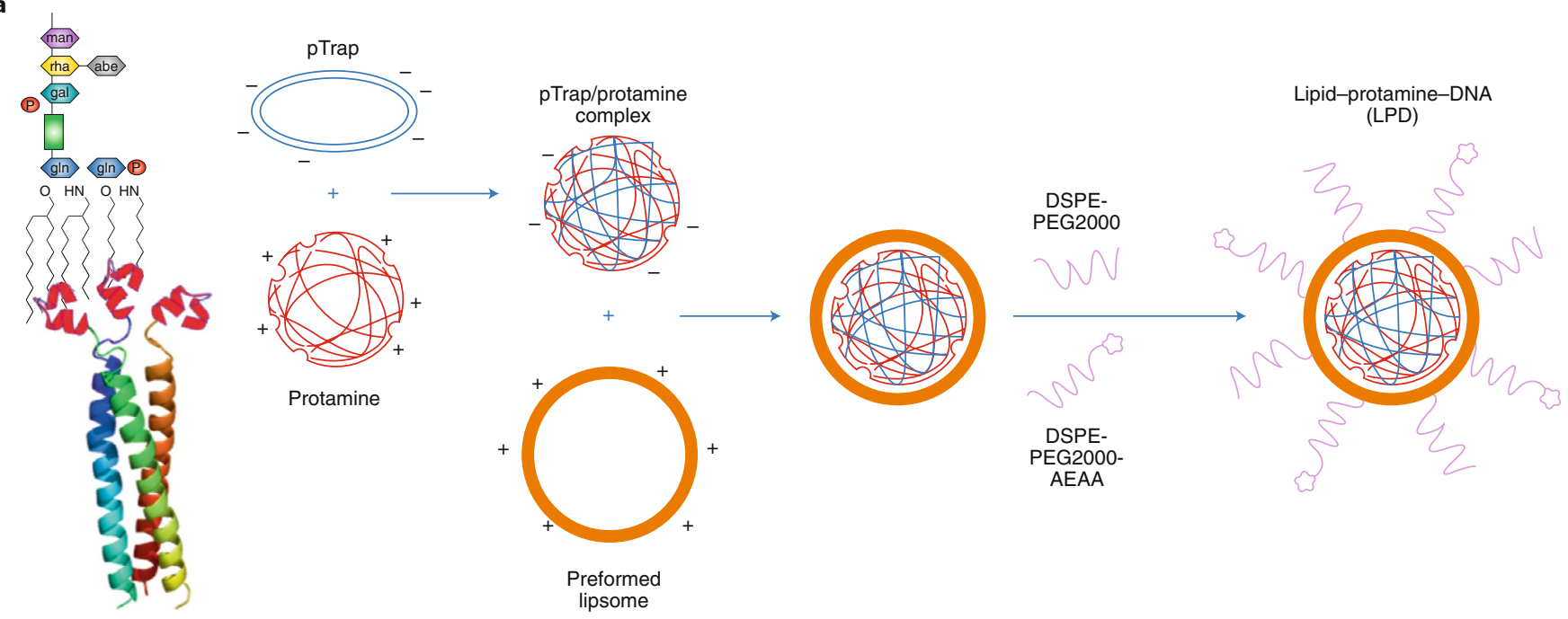

b



Fig. 4 | Examples of nanotechnology that manipulate or respond to microbiome signals/metabolites. a, An LPS trap, interaction of trap with LPS (red binding regions) and formulation of trap plasmid-loaded lipid nanoparticles. AEAA, N-(2-aminoethyl)-4-methoxybenzamide. PEG, polyethylene glycol. DSPE, 1,2-Distearoylphosphatidylethanolamine. pTrap, trap plasmid. b. Doxorubicin release from a triple-layered nanogel in response to local presence of bacteria-secreted lipase in the tumour microenvironment for tumour cell killing. Reproduced with permission from ref. ${ }^{51}$, Wiley (a) and reprinted with permission from ref. ${ }^{57}$, American Chemical Society (b).

of bacteria and thus they typically act as broad-spectrum antimicrobials. Targeted delivery of antimicrobials to specific bacteria species implicated in gut cancers (for example, H. pylori, F. nucleatum) (Fig. 5a) can potentially address the negative side effects to the commensal microbiota that is usually associated with broad-spectrum antibiotics. Extending beyond the gut, nanotechnologies designed to deliver antimicrobials can be extended to kill TAB at distal sites of metastasis via systemic administration (Fig. 5b). Nanotechnologies can also be used to deliver prebiotics, which are food-based compounds that selectively stimulate growth or activity of individual or groups of bacterial species ${ }^{69}$. Towards anti-cancer effects, nanotechnologies can be used to formulate and deliver prebiotics that specifically regulate the proliferation or metabolism of commensal bacteria with known anti-cancer effects ${ }^{58,59}$ (Fig. 5a). Strategies for nanotechnology-based prebiotic systems can potentially target delivery of prebiotics to specific microbial niches in the gut by using stimuli-responsive (for example, $\mathrm{pH}$, bacterial metabolites) release mechanisms or targeting strategies to specific bacterial species. Here, we will discuss these two different nanotechnology strategies by first highlighting a study that used a molecular targeting approach to increase efficacy in killing cancer-causing $H$. pylori, which has potential for cancer prevention. We then highlight a second example wherein a targeted nanotechnology enables microbiome modulation through prebiotic delivery for upregulation of an anticancer commensal microbe. Finally, we discuss considerations at the forefront of microbiome research that may inform future nanotechnology design for microbiome modulation.

With the growing need for targeted antibiotics that selectively kill microbes while sparing the commensal microbiota, nanotechnologies stand out as a potential therapeutic platform. Nanotechnologies are amenable to surface modifications for targeted delivery, can be functionalized to navigate the mucosal and epithelial barriers, and encapsulate and release antibiotics in a controlled manner. Indeed, there exists recent examples of nanotechnologies targeted to specific bacteria to facilitate antibiotic delivery ${ }^{65}$. The connection between $H$. pylori and stomach cancer described above is well known and provides a clear target for intervention as a cancer prevention strategy. A poly(lactic-co-glycolic acid) (PLGA) nanoparticle, encapsulating the antibiotic clarithromycin, was coated with gastric epithelial cell membranes (AGS cells) to bind to $H$. pylori. This approach used the existing interactions between receptors on AGS cells and adhesins on the surface of $H$. pylori that enable binding to the gastric epithelium (Fig. 6a). It was confirmed via both scanning electron microscopy and confocal microscopy that the nanoparticle formulation specifically targets $H$. pylori, unlike non-targeted nanoparticles. AGS-NPs were then evaluated in an $H$. pylori infection 
a


b
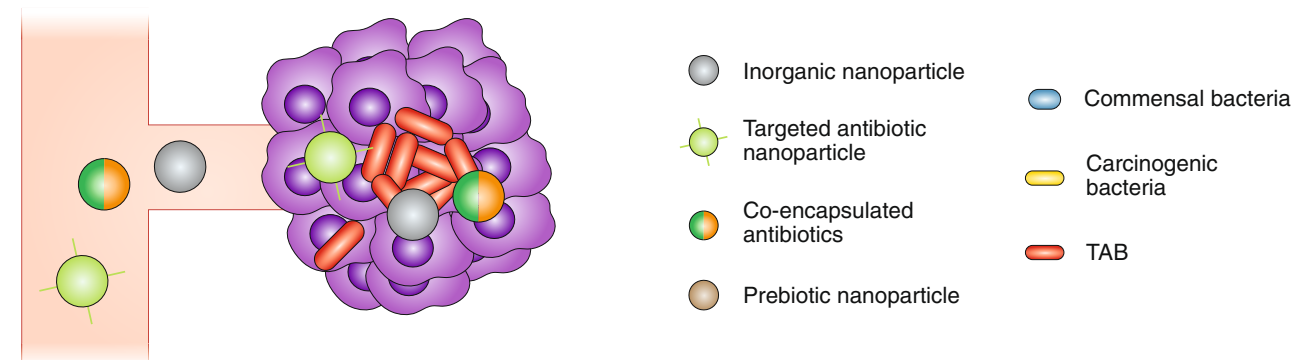

Fig. 5 | Nanotechnology strategies for microbiome modulation. a, The gut, where antimicrobial nanoparticles kill carcinogenic bacteria or prebiotic nanoparticles support proliferation of anti-cancer commensal bacteria. $\mathbf{b}$, The distal tumour microenvironment where antimicrobial nanoparticles kill TAB.

model in vivo, where targeted AGS-NPs improved $H$. pylori killing in the stomach as compared to non-targeted nanoparticles (1.4-log enhancement), free antibiotics (2-log enhancement) and a negative control (3-log enhancement ${ }^{44}$. This study highlights how wellknown functions of nanotechnologies (for example, cell-membrane coatings, encapsulation of small molecule drugs and navigating and targeting in complex microenvironments) can form the technological basis for targeted killing of cancer-causing microbes as a prevention strategy.

In more recent work, a nanotechnology was designed to improve colorectal cancer treatment and outcomes, using a multi-faceted approach (Fig. 6b). First, a bacteriophage, which are viruses that infect, propagate in, and subsequently lyse bacteria ${ }^{70}$, was isolated from human saliva and demonstrated the ability to eliminate cancer-causing F. nucleatum. The isolated bacteriophage was functionalized to facilitate click-chemistry based linkage to nanoparticles to provide enhanced nanoparticle targeting to colorectal cancer sites with F. nucleatum burden. This provided the first part of a multitiered strategy to treat colorectal cancer by targeting deletion of F. nucleatum. Bacteriophages are often highly specific and capable of eliminating single species of bacteria while sparing others; the phage isolated for this study exhibited minimal inhibition against five other bacterial strains. Importantly, one of the strains unaffected by the phage was Clostridium butyricum, which is known to secrete short-chain fatty acids that have been demonstrated to suppress growth of colorectal cancer ${ }^{58}$. As such, the material choice for the nanotechnology was selected based on the material's potential for facilitating growth of C. butyricum. Eight different types of nanoparticles, ranging from gold-based to dextran-based, were evaluated for their effect on both F. nucleatum and C. butyricum, and it was shown that dextran-based nanoparticles increased proliferation of C. butyricum by over 5-fold. It is likely that dextran played the role of a prebiotic ${ }^{69}$ by providing metabolites that facilitate growth of $C$. butyricum through nanoparticle degradation. This approach provided the second mechanism by which this multifaceted nanoparticle could improve colorectal cancer therapy by increasing the abundance of C. butyricum at the site of colorectal cancer. Finally, irinotecan, a clinically used chemotherapy against colorectal cancer, was encapsulated in dextran nanoparticles to provide a third and distinct mechanism to kill colorectal cancer cells.
It was shown that sequential delivery of phage (functionalized to facilitate click-chemistry based linkage to nanoparticles), followed by nanoparticles led to over 3-fold increase in nanoparticle accumulation in colorectal cancer tumours. In a therapeutic model against colorectal cancer, the combined therapy included phage, the prebiotic dextran nanoparticle, and encapsulated irinotecan to provide significant survival advantages as compared to individual groups consisting of antibiotics, irinotecan, and non-targeted nanoparticles. Furthermore, it was confirmed that the dextran-based nanoparticle increased abundance of commensal strains such as C. butyricum, while reducing $F$. nucleatum burden ${ }^{71}$. This work demonstrated nanotechnology-mediated efficacy against colorectal cancer via delivery of a prebiotic and chemotherapeutic-releasing nanoparticle to simultaneously increase key commensal anti-cancer species and kill tumour cells. The phage-primed targeting approach provided a means to directly target nanoparticles to $F$. nucleatum burdened tumours while selectively killing cancer-causing $F$. nucleatum. Importantly, the authors isolated and identified the phage as part of this work, potentially laying the outline for a possible workflow in developing personalized nanoparticle therapies against TAB.

The first example highlights a preventative application where a targeted nanotechnology improved killing of cancer-causing bacteria before a tumour is formed. The second example highlights a therapeutic application where existing TAB were killed by a phage-guided targeting approach for nanoparticles; this study also highlighted how a targeted approach could be used to modulate the existing microbiota towards an anti-cancer state for improved tumour killing. This study highlighted how the commensal microbiota can be used for anti-cancer function. In addition to utilizing C. butyricum to aid in cancer cell killing, other species of the commensal microbiota are known to play a large role in immunotherapy efficacy ${ }^{11,12}$ and in governing inflammation or dysbiosis, both of which are separately linked to cancer ${ }^{62,63}$. Moving forward, understanding and subsequently mitigating unintended damage to the commensal microbiota from targeted nanotechnologies will be essential. These studies will be most informative if they can identify nanotechnology properties that enable high killing of the targeted bacteria whilst minimizing changes to the commensal microbiota, or, as shown here, promoting beneficial commensal species. Beyond opportunities for targeted deletion, nanotechnologies can provide functions that could be used 


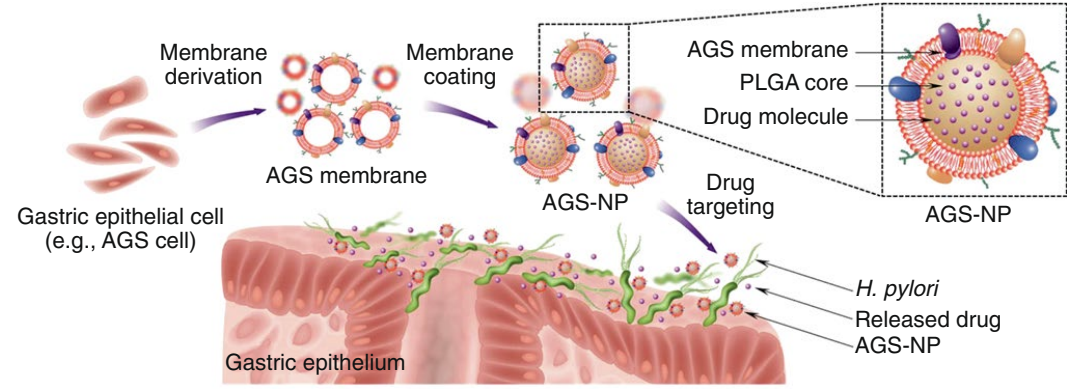

b

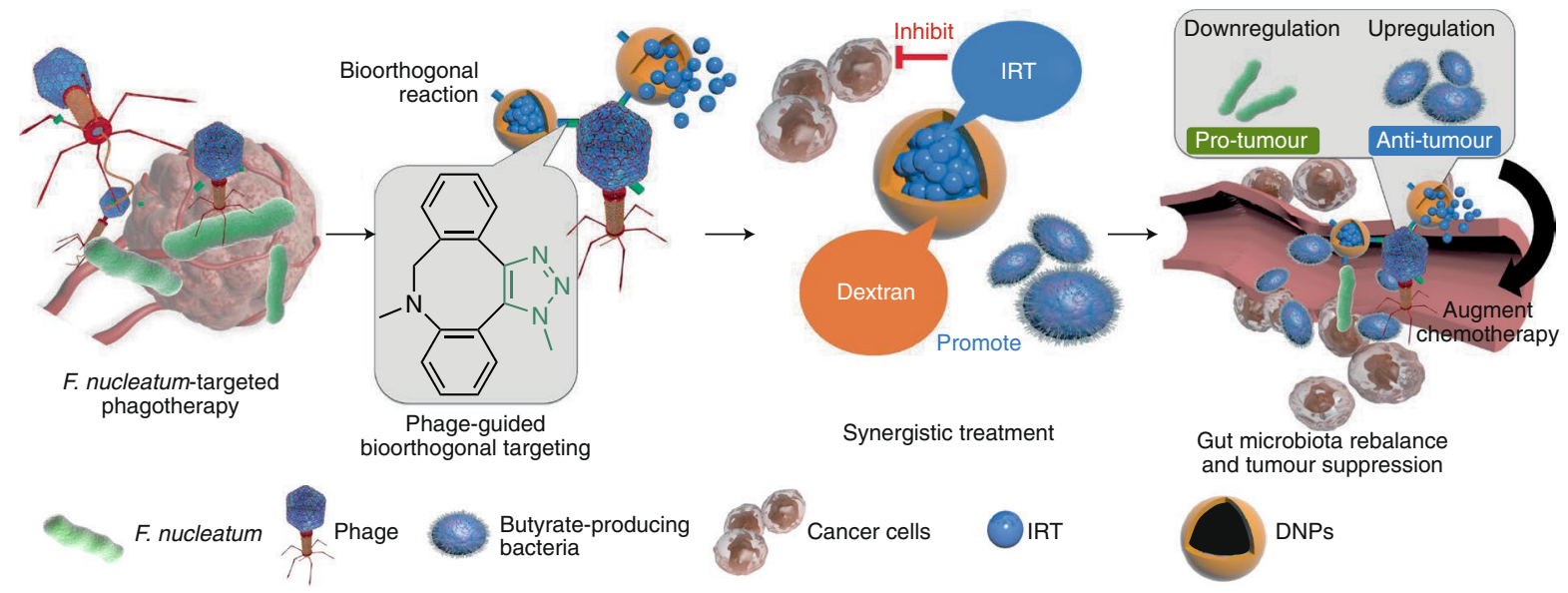

Fig. 6 | Examples of nanotechnologies used for microbiome modulation. a, The preparation of epithelial cell membrane-coated and antibiotic-loaded PLGA nanoparticles for targeting and killing of $H$. pylori. $\mathbf{b}$, Multi-faceted nanotechnology that leverages phage-based targeting to deliver prebiotics to upregulate anti-cancer commensal bacteria and chemotherapeutics to kill cancer cells in TAB-burdened tumours. IRT, irinotecan. DNPs, dextran nanoparticles. Reproduced with permission from ref. ${ }^{44}$, Wiley (a) and reprinted by permission from ref. ${ }^{71}$, Springer Nature Ltd (b).

to address the growing concerns of antimicrobial resistance ${ }^{60}$ that exist for cancer-causing bacteria such as H. pylori ${ }^{72}$ and F. nucleatum ${ }^{73}$, often resulting from exposure to traditional antibiotics ${ }^{74}$. As such, efforts to develop antimicrobial approaches that complement or synergize with antibiotics through distinct mechanisms of action may be able to address concerns with antimicrobial resistance if complete eradication can be achieved. For example, inorganic nanoparticles kill bacteria primarily via reactive oxygen species $^{68}$, which may synergize with antibiotics to enhance killing of cancer-causing bacteria ${ }^{75}$. In other approaches, nanotechnology-mediated delivery of combinations of antibiotics has been leveraged to kill $H$. pylori in higher amounts than individual antibiotics alone. By delivering a combination of amoxicillin, clarithromycin and omeprazole in a single a chitosan-based nanoparticle, $H$. pylori burden in mice was eliminated in higher amounts as compared to the individual nanoparticle antibiotic formulations ${ }^{64}$. These efforts collectively highlight the potential of nanotechnologies in killing specific cancer-causing bacteria and how they may address antimicrobial resistance concerns.

\section{Microbe-inspired nanotechnologies}

Individual bacteria possess the abilities to navigate complex environments and negotiate biological barriers, unlike many synthetic nano/microscale technologies; for example, certain bacteria can actively migrate towards chemical gradients ${ }^{76}$ or penetrate biological barriers ${ }^{77}$. One well-described phenomena is the accumulation of intravenously injected bacteria to hypoxic areas of tumours and their subsequent colonization at the tumour site as $\mathrm{TAB}^{78}$. By attaching or hitchhiking nanoparticles to the surface of bacteria, numerous approaches to improve nanoparticle delivery to tumours or other tissues have been described (Fig. 7). A few notable examples

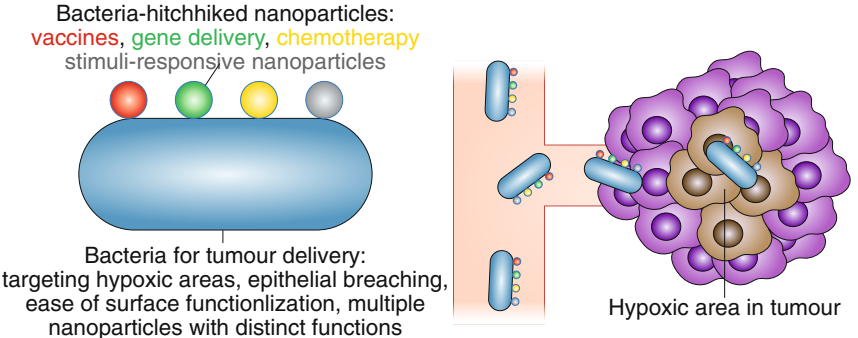

Fig. 7 | Microbe-inspired nanotechnologies. Schematic showing bacteriahitchhiked nanoparticles for cancer treatment, their potential applications and their affinity for hypoxic areas in tumours.

include using bacteria-nanotechnology complexes to facilitate in vivo gene editing of mammalian cells ${ }^{79}$, oral therapeutic cancer vaccination $^{80}$ and on-demand local delivery of drugs to tumours ${ }^{81}$. Strategies that combine bacterial carriers and nanoparticles payloads have been reviewed extensively elsewhere ${ }^{82}$, and will not be covered in depth here.

In contrast to the above studies where the natural functions of bacteria were used to facilitate nanoparticle delivery, a new approach using the existing physiology of bacteria to facilitate nanoparticle synthesis in vivo to improve oral drug delivery was developed. The probiotic and spore-forming bacteria Bacillus coagulans was used as a factory for nanoparticle synthesis and delivery to treat colon cancer. B. coagulans spores are comprised of a hydrophobic protein coating that is capable of resisting harsh conditions including stomach acids, while allowing shedding and germination in favourable 

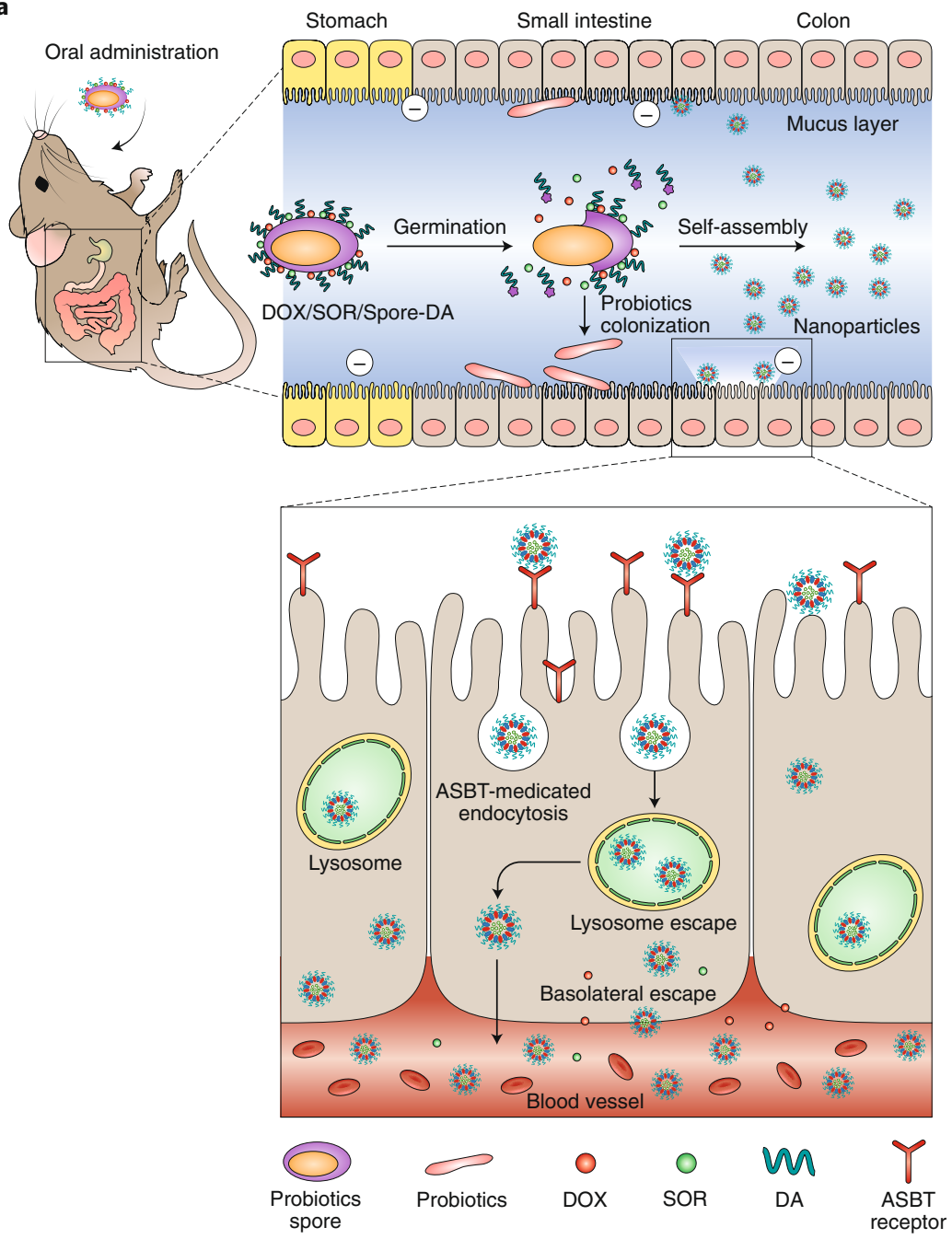

b
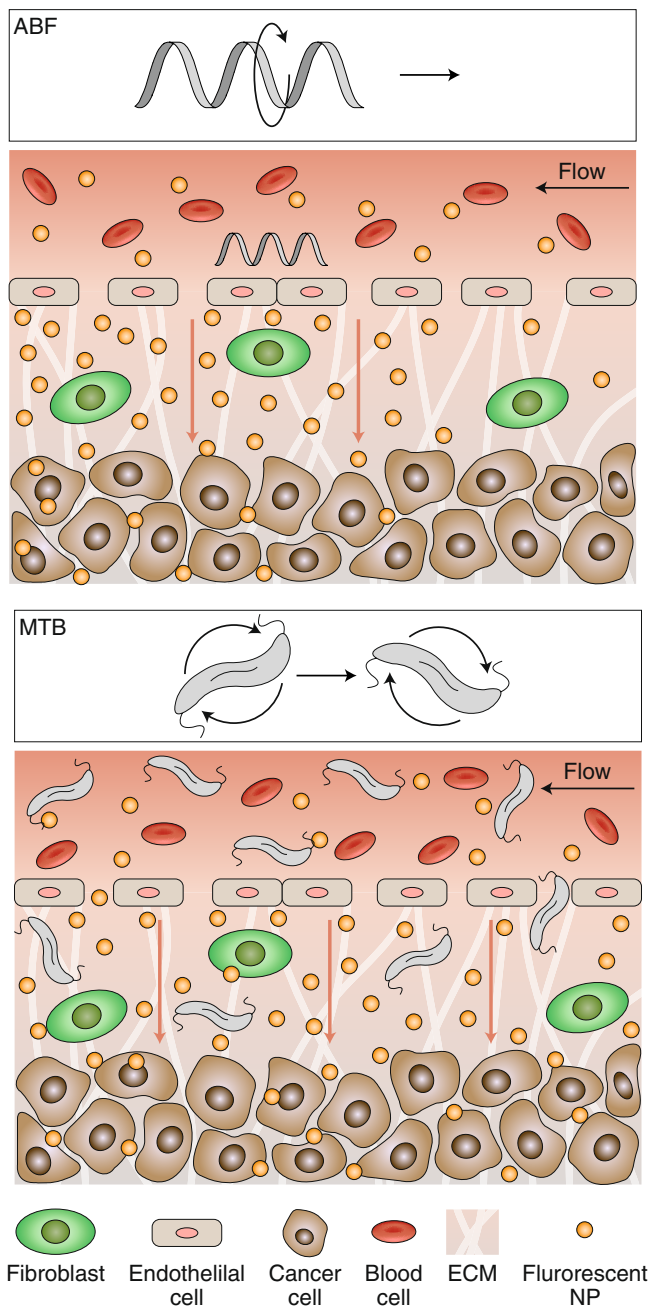

Fig. 8 | Examples of microbe- or microbiome-inspired nanotechnologies. a, Schematic of the spore-based nanoparticle generator approach used for colon cancer treatment. ASBT, apical sodium-dependent bile acid transporter. DA, deoxycholic acid. DOX, doxorubicin. SOR, sorafenib. b, A synthetic microfabricated technology (artificial bacterial flagellum, ABF) or swarms of magnetotactic bacteria (MTB) were used to introduce convective flow. Reproduced with permission from ref. ${ }^{83}$, Wiley (a) and reprinted from ref. ${ }^{84}$, AAAS (b).

environments such as the intestines. It was hypothesized that hydrophilic modification of the spore exterior would lead to autonomous nanoparticle generation via self-assembly upon shedding of the hydrophobic spore coating (Fig. 8a). B. coagulans were first induced towards spore formation, then surface conjugated with deoxycholic acid to increase epithelial transport or hyaluronic acid to enable mucoadhesion, and finally doxorubicin and sorafenib were passively adsorbed to the spore-deoxycholic acid or spore-hyaluronic acid complexes. It was demonstrated that the spore-deoxycholic acid formulations exhibited higher transport through a Caco-2 monolayer in vitro and facilitated higher drug absorption in vivo in rats after oral administration as compared to free drug controls. The hyaluronic acid formulation successfully generated observable nanoparticles in the intestines in vivo, and outperformed free drugs in preventing growth of a colon cancer tumour in vivo ${ }^{83}$. Oral delivery of chemotherapeutics in clinical settings could improve patient acceptance, especially for orally accessible primary tumours in the colon. This work used the natural physiology of spore forming bacteria to enable the oral delivery of chemotherapeutics. By virtue of spores germinating in favouable environments, nanoparticle generation and eventual drug release could be localized downstream of the stomach and upstream of the site of colon cancer.
Unlike functions that individual bacteria perform, some phenomena (for example, quorum sensing) only arise from the interplay and collective behaviour of microbe communities or microbiomes. Few complex behaviours of microbe communities are well understood, and as such, opportunities in recreating these potentially useful functions have yet to be widely explored. Here, we will highlight a recent approach that mimics the collective behaviour of microbe communities to improve traditional nanoparticle delivery across fenestrated barriers. Nanoparticles are known to exhibit enhanced tumour accumulation via the passive EPR effect, which is dominantly dependent on nanoparticle diffusion. Complementary to diffusion-mediated transport, introduction of convective flow could further enhance tumour accumulation of nanoparticles. In this work, a living microbe community of magnetotactic bacteria (MTB) were exposed to an externally applied magnetic field to induce convective flow in microchannels. The addition of convective flow improved transport of nanoparticles across microchannel pores as compared to controls without convective flow. Inspired by the collective behaviour of MTB, a synthetic microfabricated technology (artificial bacterial flagellum; ABF) (Fig. 8b) was developed as a synthetic technology to induce convective flow and improve nanoparticle transport. It was demonstrated that the convective 
flow introduced by the $\mathrm{ABF}$ enhanced nanoparticle transport across $50 \mu \mathrm{m}$ openings as compared to microchannels without the ABF. Likewise, for the MTB community, a rotating magnetic field (RMF) enhanced nanoparticle transport across the same pores as compared to the MTB without the $\mathrm{RMF}^{84}$. The clinical implementation of nanotechnologies has faced considerable challenges, including addressing the low dose delivered to tumours ${ }^{85}$. This work, inspired by the collective behaviour of a microbe-community, provides a new microbe-inspired strategy to improve traditional nanoparticle transport across pores ${ }^{84}$. Broadly, both the synthetic and microbecommunity approaches provided new insights as to how convective flow can be used to enhance nanoparticle transport. The potential connections between the microbiome, cancer and nanotechnology extend beyond the use of nanotechnologies in modulating the microbiome and, if understood, can be used to motivate the design of new technologies to improve delivery of traditional nanoparticles.

\section{Opportunities, challenges and perspective}

As opportunities to use nanotechnologies at the microbiome and cancer interface emerge, key challenges that arise from the microbiome, the tumour location and microenvironment, and the limitations of nanotechnologies will need to be addressed. For example, the balance of microbes in the microbiome and their associated feedback loops are delicate and dynamic ecosystems that have considerable implications in cancer through dysbiosis- or inflammation-mediated mechanisms $\mathrm{s}^{62,63}$ or in mediating efficacy of various cancer therapies ${ }^{10-12}$. As nanotechnology-based microbiome interventions are developed and evaluated, future work should study toxicity, side effects and downstream effects as they relate to the commensal microbiome given the implications for cancer development, progression and treatment. Initial work that has studied nanotechnology effects on the commensal microbiota has been predominately focused on inorganic nanoparticles (for example, silver, zinc, titanium and silica) ${ }^{86,87}$ given their prevalence and use in many consumer products ${ }^{86,88}$. The effects of inorganic nanotechnologies on the microbiome have been previously reviewed ${ }^{86}$ and current evidence suggests that nanotechnology properties, the local microenvironment and the existing composition of the microbiota can affect how the microbiota changes upon exposure to inorganic nanoparticles $^{86}$, indicating a complex relationship. It has been postulated that exposure of the commensal microbiota to nanotechnologies could potentially lead to microbial imbalance or dysbiosis ${ }^{89}$. As such, the effects of nanotechnologies on the microbiota must be studied in more depth and expanded beyond inorganic nanotechnologies, so as to better inform the design and implementation of nanotechnologies for intervention of the microbiome for cancer therapy.

Since the microbiome is a patient-unique ecosystem that dynamically changes in response to diet ${ }^{90}$, drugs ${ }^{91}$ and other external factors, nanotechnologies must be capable of performing their delivery, therapeutic, and microbiome modulating functions under a variety of changing microbiome conditions and states (that is, different locations, populations and concentrations of microbes). Since these factors cannot currently be predicted in vivo or in the clinic, the initial uses of nanotechnology in microbiome intervention have focused on targeting specific microbes or metabolites with known roles in carcinogenesis. However, in clinical practice these microbes and/or metabolites must first be identified prior to intervention. This points towards an unmet need, and diagnostic challenge, in locating and identifying cancer-causing microbes, which can be found in the gut or in distal tissues as TABs, and/or their metabolites in a patient. While this Review focused on nanotechnology interventions of the microbiome, there are clear opportunities and motivations for using nanotechnologies for in vivo detection of carcinogenic microbes or their metabolites to inform therapeutic application. This can be initially approached by combining past nanotechnology efforts in imaging, diagnostics and detection of tumours and cancer cells ${ }^{92}$ or pathogenic microbes ${ }^{93}$ with novel approaches to target carcinogenic bacteria in vivo. Early detection of cancer-causing bacteria may even reveal opportunities in using nanotechnologies as a prophylactic for preventing cancer or preventing the colonization of cancer-causing bacteria.

Nanotechnologies for microbiome intervention will also face the same challenges as current cancer nanotechnologies such as scale-up, targeting efficiency and biodistribution, toxicity and side effects, and overcoming delivery challenges arising from tumour heterogeneity (for example, vascularity) between patients ${ }^{24}$. Many of these factors are governed by nanoparticle design criteria (for example, material choice and payload) and their properties (for example, size, shape, elasticity and surface charge). Understanding how these parameters affect and control nanoparticle interactions with both target and non-target cells represents a highly active area of nanotechnology research; however, efforts to describe interactions between nanoparticle properties and microbes are considerably lacking in comparison. As nanotechnologies are employed for microbiome applications, we envision the emergence of a subfield focused on understanding the effects of common nanoparticle materials and how nanoparticle properties dictate the fate and function of commensal and carcinogenic bacteria. This will lead to new, microbiome focused additions to the nanotechnology toolbox which may one day be leveraged piecewise to design nanotechnologies for specific microbiome interventions.

The nanotechnologies highlighted in this Review apply an existing and established toolbox of nanoparticle functionalities (Fig. 2) towards modulating the microbiome-tumour microenvironment (Fig. 1) to improve or enable cancer therapy via interfering with bacteria signals or using bacterial metabolites (Fig. 3), modulating the microbiome or targeted killing of TAB (Fig. 5), and in extracting inspiration from the microbial world to better design nanoparticle delivery systems (Fig. 7). In the first study we highlighted (Fig. 4a), nanotechnologies were leveraged for their well-established application of mammalian cell transfection to genetically engineer the microbiome-tumour microenvironment to block microbiome metabolites from interacting with cancer cells. In the second study we discussed (Fig. 4b), specific metabolites secreted by TAB initiated a release of an anti-cancer drug from stimuli-responsive nanotechnology. In the third highlighted case (Fig. 6a), targeting ligands and bioinspired cell-membrane coatings were used to functionalize the surface of antibiotic-loaded nanoparticles to enable targeting and killing of specific bacteria. Importantly, in these applications, nanotechnologies were able to address multi-scale challenges in the vascular system, the tumour microenvironment and in the gut by leveraging an already-existing, and ever-expanding, nanotechnology toolbox. This existing nanotechnology toolbox exists because of the decades of research efforts that have focused on preclinical and clinical development of cancer nanomedicines. We then highlighted a recent example (Fig. 6b) where TAB were targeted with bacteriophage to both locally kill the TAB and act as a molecular anchor for a subsequently delivered nanoparticle. This phage-guided nanoparticle then delivered chemotherapeutics to the tumour site and delivered prebiotics to promote a beneficial commensal microbiota species that directly inhibits cancer cell growth. The next paper we highlighted in this Review (Fig. 8a) added a new component to the existing nanotechnology toolbox through utilizing bacterial spores as a factory for nanoparticle synthesis in vivo and as a vehicle for local delivery to tumours in the gut. The final paper we highlight (Fig. 8b) used a basic understanding of phenomena that only occur under specific conditions with microbe communities to improve nanoparticle delivery. Recreating bacterial phenomena with a synthetic technology to improve traditional nanoparticle delivery highlights a strong reciprocal relationship at the intersection of microbiome, cancer and nanotechnology research. As the role of the microbiome in cancer and other diseases (for example, autoimmune, neurological and inflammatory) 
are discovered and described ${ }^{94-98}$, efforts perturbing the microbiome to understand the associated implications on disease and treatment may result in the identification of additional microbe targets or opportunities for treatment of other diseases. As an example, a recent paper described a hyaluronic acid-bilirubin nanotechnology that could target inflamed colon tissue, increase the diversity and richness of the microbiome, restore epithelial barrier properties through upregulating tight-junction proteins and treat colitis ${ }^{99}$. The development of nanotechnologies designed to navigate and negotiate the microbiome could apply to these other diseased states as well, provided that individual microbes or the balance of microbes are implicated. This is partly because the biological barriers and physiological challenges remain relatively conserved, and in cases where they are not, nanotechnologies have been leveraged as a tunable platform technology for decades and could be modified as needed for specific barriers and challenges. Overall, the use of nanotechnologies in microbiome modulation for cancer applications is still a nascent field; however, these studies highlight the potential that exists at this expanding intersection.

Received: 31 May 2019; Accepted: 6 November 2019; Published online: 4 December 2019

\section{References}

1. Turnbaugh, P. J. et al. The human microbiome project. Nature 449, 804 (2007)

2. Helmink, B. A., Khan, M. A. W., Hermann, A., Gopalakrishnan, V. \& Wargo, J. A. The microbiome, cancer, and cancer therapy. Nat. Med. 25, 377-388 (2019). A recent review discussing the influence of gut microbiota on cancer therapy and current approaches targeting gut microbiome for cancer therapy.

3. Schwabe, R. F. \& Jobin, C. The microbiome and cancer. Nat. Rev. Cancer 13, 800 (2013)

An article that summarizes the links between bacterial microbiota and cancer, many of the driving mechanisms, and strategies that involve targeting the microbiome for cancer prevention.

4. Hatakeyama, M. Oncogenic mechanisms of the Helicobacter pylori CagA protein. Nat. Rev. Cancer 4, 688 (2004).

5. Rubinstein, M. R. et al. Fusobacterium nucleatum promotes colorectal carcinogenesis by modulating E-cadherin/beta-catenin signaling via its FadA adhesin. Cell Host Microbe 14, 195-206 (2013).

6. Arthur, J. C. et al. Intestinal inflammation targets cancer-inducing activity of the microbiota. Science 338, 120-123 (2012).

7. Bhatt, A. P., Redinbo, M. R. \& Bultman, S. J. The role of the microbiome in cancer development and therapy. CA Cancer J. Clin. 67, 326-344 (2017).

8. Bullman, S. et al. Analysis of Fusobacterium persistence and antibiotic response in colorectal cancer. Science 358, 1443-1448 (2017).

This paper demonstrated that Fusobacterium and its associated microbiome is maintained in distal metastases and motivates the need to target $\mathrm{TAB}$.

9. Riquelme, E. et al. Tumor microbiome diversity and composition influence pancreatic cancer outcomes. Cell 178, 795-806e712 (2019).

10. Viaud, S. et al. The intestinal microbiota modulates the anticancer immune effects of cyclophosphamide. Science 342, 971-976 (2013).

11. Iida, N. et al. Commensal bacteria control cancer response to therapy by modulating the tumor microenvironment. Science 342, 967-970 (2013).

12. Derosa, L. et al. Negative association of antibiotics on clinical activity of immune checkpoint inhibitors in patients with advanced renal cell and non-small-cell lung cancer. Ann. Oncol. 29, 1437-1444 (2018).

13. Vetizou, M. et al. Anticancer immunotherapy by CTLA-4 blockade relies on the gut microbiota. Science 350, 1079-1084 (2015).

14. Sivan, A. et al. Commensal Bifidobacterium promotes antitumor immunity and facilitates anti-PD-L1 efficacy. Science 350, 1084-1089 (2015).

15. Tanoue, T. et al. A defined commensal consortium elicits CD8 T cells and anti-cancer immunity. Nature 565, 600-605 (2019).

16. Routy, B. et al. Gut microbiome influences efficacy of PD-1-based immunotherapy against epithelial tumors. Science 359, 91-97 (2018).

17. Geller, L. T. et al. Potential role of intratumour bacteria in mediating tumor resistance to the chemotherapeutic drug gemcitabine. Science 357, $1156-1160$ (2017).

18. McQuade, J. L., Daniel, C. R., Helmink, B. A. \& Wargo, J. A. Modulating the microbiome to improve therapeutic response in cancer. Lancet Oncol. 20, e7-e91 (2019).

19. Gopalakrishnan, V., Helmink, B. A., Spencer, C. N., Reuben, A. \& Wargo, J. A. The influence of the gut microbiome on cancer, immunity, and cancer immunotherapy. Cancer cell 33, 570-580 (2018).
20. Young, V. B. Therapeutic manipulation of the microbiota: past, present, and considerations for the future. Clin. Microbiol. Infect. 22, 905-909 (2016).

21. Vargason, A. M. \& Anselmo, A. C. Clinical translation of microbe-based therapies: Current clinical landscape and preclinical outlook. Bioeng. Transl. Med. 3, 124-137 (2018).

22. Mimee, M., Citorik, R. J. \& Lu, T. K. Microbiome therapeutics-advances and challenges. Adv. Drug Deliv. Rev. 105, 44-54 (2016).

23. Jobin, C. Precision medicine using microbiota. Science 359, 32-34 (2018).

24. Anselmo, A. C. \& Mitragotri, S. Nanoparticles in the clinic. Bioeng. Transl. Med. 1, 10-29 (2016).

25. Li, S.-D. \& Huang, L. Pharmacokinetics and biodistribution of nanoparticles Mol. Pharmaceut. 5, 496-504 (2008).

26. Owens, D. E. III \& Peppas, N. A. Opsonization, biodistribution, and pharmacokinetics of polymeric nanoparticles. Int. J. Pharm. 307, 93-102 (2006).

27. Matsumura, Y. \& Maeda, H. A new concept for macromolecular therapeutics in cancer chemotherapy: mechanism of tumoritropic accumulation of proteins and the antitumor agent smancs. Cancer Res. 46, 6387-6392 (1986).

28. Kamaly, N., Yameen, B., Wu, J. \& Farokhzad, O. C. Degradable controlledrelease polymers and polymeric nanoparticles: mechanisms of controlling drug release. Chem. Rev. 116, 2602-2663 (2016).

29. Anselmo, A. C. \& Mitragotri, S. Nanoparticles in the clinic: An update. Bioeng. Transl. Med. 4, e10143 (2019).

30. Peer, D. et al. Nanocarriers as an emerging platform for cancer therapy. Nature Nanotechnol. 2, 751-760 (2007).

31. Ma, L., Kohli, M. \& Smith, A. Nanoparticles for combination drug therapy. ACS nano 7, 9518-9525 (2013).

32. Mura, S., Nicolas, J. \& Couvreur, P. Stimuli-responsive nanocarriers for drug delivery. Nat. Mater. 12, 991-1003 (2013).

33. Moon, J. J., Huang, B. \& Irvine, D. J. Engineering nano-and microparticles to tune immunity. Adv. Mater. 24, 3724-3746 (2012).

34. Blanco, E., Shen, H. \& Ferrari, M. Principles of nanoparticle design for overcoming biological barriers to drug delivery. Nature Biotechnol. 33, 941-951 (2015).

35. Hu, C. M. et al. Nanoparticle biointerfacing by platelet membrane cloaking. Nature 526, 118-121 (2015).

36. Rodriguez, P. L. et al. Minimal "self" peptides that inhibit phagocytic clearance and enhance delivery of nanoparticles. Science 339, 971-975 (2013).

37. Petros, R. A. \& DeSimone, J. M. Strategies in the design of nanoparticles for therapeutic applications. Nat. Rev. Drug Discov. 9, 615-627 (2010).

38. Albanese, A., Tang, P. S. \& Chan, W. C. The effect of nanoparticle size, shape, and surface chemistry on biological systems. Annu. Rev. Biomed. Eng. 14, 1-16 (2012).

39. Bertrand, N., Wu, J., Xu, X., Kamaly, N. \& Farokhzad, O. C. Cancer nanotechnology: the impact of passive and active targeting in the era of modern cancer biology. Adv. Drug Deliv. Rev. 66, 2-25 (2014).

40. Tropini, C., Earle, K. A., Huang, K. C. \& Sonnenburg, J. L. The gut microbiome: connecting spatial organization to function. Cell Host Microbe 21, 433-442 (2017).

41. Hua, S., Marks, E., Schneider, J. J. \& Keely, S. Advances in oral nano-delivery systems for colon targeted drug delivery in inflammatory bowel disease: Selective targeting to diseased versus healthy tissue. Nanomedicine: NBM 11, 1117-1132 (2015).

42. Jin, K., Luo, Z., Zhang, B. \& Pang, Z. Biomimetic nanoparticles for inflammation targeting. Acta Pharm. Sin. B 8, 23-33 (2018).

43. Lai, S. K., Wang, Y.-Y. \& Hanes, J. Mucus-penetrating nanoparticles for drug and gene delivery to mucosal tissues. Adv. Drug Deliv. Rev. 61, 158-171 (2009).

44. Angsantikul, P. et al. Coating nanoparticles with gastric epithelial cell membrane for targeted antibiotic delivery against Helicobacter pylori infection. Adv. Ther. (Weinh) 1, 1800016 (2018).

This manuscript describes a strategy that facilitates antibiotic delivery to specific bacteria via a targeted nanotechnology.

45. Pridgen, E. M. et al. Transepithelial transport of Fc-targeted nanoparticles by the neonatal fc receptor for oral delivery. Sci. Transl. Med. 5, 213 ra167 (2013).

46. Brown, J. M. \& Wilson, W. R. Exploiting tumour hypoxia in cancer treatment. Nat. Rev. Cancer 4, 437-447 (2004).

47. Poon, Z., Chang, D., Zhao, X. \& Hammond, P. T. Layer-by-layer nanoparticles with a $\mathrm{pH}$-sheddable layer for in vivo targeting of tumour hypoxia. ACS nano 5, 4284-4292 (2011).

48. Song, W. et al. Synergistic and low adverse effect cancer immunotherapy by immunogenic chemotherapy and locally expressed PD-L1 trap. Nat. Commun. 9, 2237 (2018)

49. Hu, S. et al. The microbe-derived short chain fatty acid butyrate targets miRNA-dependent p21 gene expression in human colon cancer. PloS one 6 , e16221 (2011).

50. Postler, T. S. \& Ghosh, S. Understanding the holobiont: how microbial metabolites affect human health and shape the immune system. Cell Metab. 26, 110-130 (2017). 
A comprehensive review that discusses how microbial metabolites affect the host immune system, thereby highlighting a multitude of targets for manipulation of microbiome signals.

51. Song, W. T. et al. Trapping of Lipopolysaccharide to Promote Immunotherapy against Colorectal Cancer and Attenuate Liver Metastasis. Adv. Mater. 30, 1805007 (2018).

In this study, a nanotechnology was used to block signals from the gut microbiome to facilitate and enhance cancer immunotherapy.

52. Dapito, D. H. et al. Promotion of hepatocellular carcinoma by the intestinal microbiota and TLR4. Cancer cell 21, 504-516 (2012).

53. Goodwin, T. J., Zhou, Y., Musetti, S. N., Liu, R. \& Huang, L. Local and transient gene expression primes the liver to resist cancer metastasis. Sci. Transl. Med. 8, 364ra153 (2016).

54. Kostic, A. D. et al. Fusobacterium nucleatum potentiates intestinal tumorigenesis and modulates the tumor-immune microenvironment. Cell Host Microbe 14, 207-215 (2013).

55. Pushalkar, S. et al. The pancreatic cancer microbiome promotes oncogenesis by induction of innate and adaptive immune suppression. Cancer Discov. 8 , 403-416 (2018).

56. Yu, T. et al. Fusobacterium nucleatum promotes chemoresistance to colorectal cancer by modulating autophagy. Cell 170, 548-563 (2017).

57. Xiong, M.-H. et al. Differential anticancer drug delivery with a nanogel sensitive to bacteria-accumulated tumor artificial environment. ACS Nano 7, 10636-10645 (2013).

This study provides an approach for using bacterial metabolism in TAB as a signal for triggering drug release from nanoparticles for selectively killing cancer cells.

58. Singh, N. et al. Activation of Gpr109a, receptor for niacin and the commensal metabolite butyrate, suppresses colonic inflammation and carcinogenesis. Immunity 40, 128-139 (2014).

59. Wang, T. et al. Structural segregation of gut microbiota between colorectal cancer patients and healthy volunteers. ISME J. 6, 320 (2012).

60. Antimicrobial Resistance: Global Report on Surveillance (World Health Organization, 2014).

61. Vangay, P., Ward, T., Gerber, J. S. \& Knights, D. Antibiotics, pediatric dysbiosis, and disease. Cell Host Microbe 17, 553-564 (2015).

62. Francescone, R., Hou, V. \& Grivennikov, S. I. Microbiome, inflammation and cancer. Cancer J. 20, 181-189 (2014).

63. $\mathrm{Wu}, \mathrm{N}$. et al. Dysbiosis signature of fecal microbiota in colorectal cancer patients. Microb. Ecol. 66, 462-470 (2013).

64. Ramteke, S., Ganesh, N., Bhattacharya, S. \& Jain, N. K. Amoxicillin, clarithromycin, and omeprazole based targeted nanoparticles for the treatment of H. pylori. J. Drug Target 17, 225-234 (2009).

65. Gao, W., Thamphiwatana, S., Angsantikul, P. \& Zhang, L. Nanoparticle approaches against bacterial infections. Wiley Interdiscip. Rev. Nanomed. Nanobiotechnol. 6, 532-547 (2014).

66. Vargas-Reus, M. A., Memarzadeh, K., Huang, J., Ren, G. G. \& Allaker, R. P. Antimicrobial activity of nanoparticulate metal oxides against peri-implantitis pathogens. Int. J. Antimicrob. Agents 40, 135-139 (2012).

67. Lu, Z., Rong, K., Li, J., Yang, H. \& Chen, R. Size-dependent antibacterial activities of silver nanoparticles against oral anaerobic pathogenic bacteria. J. Mater. Sci. Mater. Med. 24, 1465-1471 (2013).

68. Hajipour, M. J. et al. Antibacterial properties of nanoparticles. Trends Biotechnol. 30, 499-511 (2012).

69. Roberfroid, M. et al. Prebiotic effects: metabolic and health benefits. Brit. J. Nutr. 104, S1-S63 (2010).

70. Kutter, E. et al. Phage therapy in clinical practice: treatment of human infections. Curr. Pharm. Biotechnol. 11, 69-86 (2010).

71. Zheng, D.-W. et al. Phage-guided modulation of the gut microbiota of mouse models of colorectal cancer augments their responses to chemotherapy. Nat. Biomed. Eng. 3, 717-728 (2019).

This study describes a phage-primed approach for targeting nanoparticles to microbiota burdened tumours and outlines a possible workflow in developing personalized nanotechnologies against TAB.

72. Wu, W., Yang, Y. \& Sun, G. Recent insights into antibiotic resistance in Helicobacter pylori eradication. Gastroenterol. Res. Pract. https://doi. org/10.1155/2012/723183 (2012).

73. Nyfors, S., Könönen, E., Syrjänen, R., Komulainen, E. \& Jousimies-Somer, H. Emergence of penicillin resistance among Fusobacterium nucleatum populations of commensal oral flora during early childhood. J. Antimicrob. Chemother. 51, 107-112 (2003).

74. Tenover, F. C. Mechanisms of antimicrobial resistance in bacteria. Am. J. Med. 119, S3-S10 (2006).

75. Li, P., Li, J., Wu, C., Wu, Q. \& Li, J. Synergistic antibacterial effects of $\beta$-lactam antibiotic combined with silver nanoparticles. Nanotechnology 16, 1912 (2005).

76. Alon, U., Surette, M. G., Barkai, N. \& Leibler, S. Robustness in bacterial chemotaxis. Nature 397, 168 (1999).
77. Danino, T. et al. Programmable probiotics for detection of cancer in urine. Sci. Transl. Med. 7, 289ra284 (2015).

78. Forbes, N. S. Engineering the perfect (bacterial) cancer therapy. Nat. Rev. Cancer 10, 785 (2010).

79. Akin, D. et al. Bacteria-mediated delivery of nanoparticles and cargo into cells. Nature Nanotechnol. 2, 441 (2007).

80. Hu, Q. et al. Engineering Nanoparticle-Coated Bacteria as Oral DNA Vaccines for Cancer Immunotherapy. Nano Lett. 15, 2732-2739 (2015).

81. Fan, J.-X. et al. Bacteria-mediated tumor therapy utilizing photothermallycontrolled TNF- $\alpha$ expression via oral administration. Nano Lett. 18, 2373-2380 (2018)

82. Hosseinidoust, Z. et al. Bioengineered and biohybrid bacteria-based systems for drug delivery. Adv. Drug Deliv. Rev. 106, 27-44 (2016).

83. Song, Q. et al. A probiotic spore-based oral autonomous nanoparticles generator for cancer therapy. Adv. Mater. 31, 1903793 (2019). This manuscript describes an approach utilizing natural functions of spore forming bacteria to autonomously produce nanoparticles in the intestine.

84. Schuerle, S. et al. Synthetic and living micropropellers for convectionenhanced nanoparticle transport. Sci. Adv. 5, eaav4803 (2019). This study develops a bacteria-inspired approach for improving nanoparticle delivery across tumour barriers using a microfabricated technology that mimics the collective behaviour of microbe communities.

85. Wilhelm, S. et al. Analysis of nanoparticle delivery to tumours. Nat. Rev. Mater. 1, 16014 (2016).

86. Qiu, K., Durham, P. G. \& Anselmo, A. C. Inorganic nanoparticles and the microbiome. Nano Res. 11, 4936-4954 (2018).

87. Pietroiusti, A., Magrini, A. \& Campagnolo, L. New frontiers in nanotoxicology: gut microbiota/microbiome-mediated effects of engineered nanomaterials. Toxicol. Appl. Pharmacol. 299, 90-95 (2016).

88. McClements, D. J. \& Xiao, H. Is nano safe in foods? Establishing the factors impacting the gastrointestinal fate and toxicity of organic and inorganic food-grade nanoparticles. NPJ Sci. Food 1, 6 (2017).

89. Bouwmeester, H., van der Zande, M. \& Jepson, M. A. Effects of food-borne nanomaterials on gastrointestinal tissues and microbiota. Wiley Interdiscip. Rev. Nanomed. Nanobiotechnol. 10, e1481 (2018).

90. David, L. A. et al. Diet rapidly and reproducibly alters the human gut microbiome. Nature 505, 559-563 (2014).

91. Cho, I. et al. Antibiotics in early life alter the murine colonic microbiome and adiposity. Nature 488, 621-626 (2012).

92. Weissleder, R. \& Pittet, M. J. Imaging in the era of molecular oncology. Nature 452, 580 (2008).

93. Sanvicens, N., Pastells, C., Pascual, N. \& Marco, M.-P. Nanoparticle-based biosensors for detection of pathogenic bacteria. Trends Analyt. Chem. 28, 1243-1252 (2009).

94. De Luca, F. \& Shoenfeld, Y. The microbiome in autoimmune diseases. Clin. Exp. Immunol. 195, 74-85 (2019).

95. Li, B., Selmi, C., Tang, R., Gershwin, M. E. \& Ma, X. The microbiome and autoimmunity: a paradigm from the gut-liver axis. Cell. Mol. Immunol. 15, 595-609 (2018).

96. Rogers, G. B. et al. From gut dysbiosis to altered brain function and mental illness: mechanisms and pathways. Mol. Psychiatry 21, 738-748 (2016).

97. Griffiths, J. A. \& Mazmanian, S. K. Emerging evidence linking the gut microbiome to neurologic disorders. Genome Med. 10, 98 (2018).

98. Hansen, J. J. \& Sartor, R. B. Therapeutic manipulation of the microbiome in IBD: current results and future approaches. Curr. Treat. Options. Gastroenterol. 13, 105-120 (2015).

99. Lee, Y. et al. Hyaluronic acid-bilirubin nanomedicine for targeted modulation of dysregulated intestinal barrier, microbiome and immune responses in colitis. Nat. Mater. https://doi.org/10.1038/s41563-019-0462-9 (2019).

\section{Acknowledgements}

W.S. would like to acknowledge support from the National Natural Science Foundation of China $(51673185,51973215)$. A.C.A. would like to acknowledge support from the Carolina Center of Cancer Nanotechnology Excellence (C-CCNE) Pilot Grant Program supported by the National Institutes of Health (NIH) National Cancer Institute (5U54CA198999-04). L.H. is supported by NIH grant CA198999.

\section{Competing interests}

The authors declare no competing interests.

\section{Additional information}

Correspondence should be addressed to A.C.A. or L.H.

Reprints and permissions information is available at www.nature.com/reprints.

Publisher's note Springer Nature remains neutral with regard to jurisdictional claims in published maps and institutional affiliations.

(C) Springer Nature Limited 2019 